\title{
Antioxidant capacity and phenolic content of cocoa beans
}

\begin{abstract}
This study investigated the antioxidant capacity and total phenolic content of cocoa beans from different countries, namely Malaysia, Ghana, Ivory Coast and Sulawesi. The antioxidant capacity of water and ethanolic extracts prepared from cocoa beans was measured by three different assays. To estimate the total phenolic content, the assay using Folini Ciocalteu reagent was used. The water extract showed the higher value of antioxidant activity based on b-carotene bleaching assay, while the ethanolic extract showed the highest scavenging and ferric reducing activities. Ghanaian cocoa beans showed the highest antioxidant and scavenging activities, followed by Ivory Coast, Malaysian and Sulawesian. However, Malaysian and Sulawesian beans exhibited the highest ferric reducing activity, compared to the other beans. The highest phenolic content was found in Malaysian beans, followed by Sulawesian, Ghanaian and Ivory Coast. A positive correlation existed for both ethanolic ( $\mathrm{r}=$ $0.76)$ and water extracts $(r=0.78)$ between phenolic content and ferric reducing activity. Our results showed that antioxidant capacity and phenolic content of Malaysian cocoa beans were comparable to Ghanaian, Ivory Coast, and Sulawesian beans.
\end{abstract}

Keyword: Cocoa beans; Antioxidant activity; Scavenging activity; Ferric reducing activity; Phenolic content 